\title{
EFFICACY OF MAGNESIUM SULPHATE ON MATERNAL MORBIDITY AND MORTALITY IN ECLAMPSIA
}

\author{
Nadia Arif, Raja Qaseem Ahmed, Bushra Zafar, Farrukh Shehzad \\ Combined Military Hospital Okara/National University of Medical Sciences (NUMS) Pakistan
}

\begin{abstract}
Objective: To study efficacy of Magnesium Sulphate in reducing maternal morbidity and mortality in patients presenting with eclampsia.

Study Design: Prospective observational study.

Place and Duration of Study: Gynae/Obs Department, Combined Military Hospital Bannu, from Jan 2018 Sep 2019.

Methodology: A total of 134 patients fulfilling inclusion criteria were included in the study. Patient management was multidisciplinary. Convulsions were controlled by 4gram magnesium sulphate intravenously over 20 minutes as a loading dose, followed by 1gram per hour of maintenance infusion. Blood pressure was controlled with injection Labetalol 10 milligram followed by injection Hydralazine repeated after every 20 minutes till the blood pressure was controlled.

Results: A total of 134 patients had eclampsia constituting $6.8 \%$ of obstetric admissions. Case fatality was $14(10.4 \%)$ while 120 $(89.55 \%)$ of the cases responded well to treatment and survived the event. Mean age of patients was 22 years. Thirty patients $22.4 \%$ were booked and 104 patients $77.6 \%$ were un-booked. Mostly patients $122(91 \%)$ came from rural area and only $12(9 \%)$ were from urban areas with booked pregnancies having regular antenatal visits. One hundred and twenty six (94\%) patients with eclampsia presented in antenatal period and only $8(6 \%)$ presented in post natal period. Mode of delivery was cesarean section in $87(64.9 \%)$ and 47 (35.1\%) delivered vaginally. Frequency of patient requiring ventilator support, acute renal failure, disseminated intravascular coagulation, adult respiratory distress syndrome, HELLP (hemolysis, elevated liver enzymes, low platelet) syndrome was $11(8.2 \%), 46(34.3 \%), 43(32.1 \%), 35(26.1 \%), 7(5.2 \%)$ respectively. In our study case fatality due to complications of eclampsia was $8(6 \%)$ due to pulmonary edema while $5(3.7 \%)$ was due to intra-cranial bleed.

Conclusion: Magnesium sulphate is a safe and cost effective drug in reducing maternal morbidity and mortality in patients presenting with eclampsia.
\end{abstract}

Keywords: Eclampsia, Magnesium sulphate, Maternal morbidity, Maternal mortality, Pulmonary edema.

This is an Open Access article distributed under the terms of the Creative Commons Attribution License (http://creativecommons.org/licenses/by/4.0), which permits unrestricted use, distribution, and reproduction in any medium, provided the original work is properly cited.

\section{INTRODUCTION}

Eclampsia is an acute life threatening complication of pregnancy ${ }^{1}$. It accounts for about 50,000 maternal deaths a year worldwide ${ }^{2}$. Eclampsia is a pregnancy specific multi organ disorder. The main features are hypertension, proteinuria, generalized edema and tonic clonic fits with or without coma occurring after 20 weeks of gestation till 6 weeks postpartum. The earliest evidence was recorded by Hippocrates, who identified headache, altered conscious level and fits as certain abnormal features seen in some pregnancies. Eclampsia is a Latin term, $1^{\text {st }}$ appeared in Johannes Varandaeus in treatise on gynaecology (1620).

There are variety of presentation and the classic features may not be present always. Pre-eclampsia and eclampsia occur mostly in primi-gravida ${ }^{3}$. The cause of eclampsia is a pregnancy specific multi-organ disorder involving vascular endothelial damage, intravascular

Correspondence: Dr Nadia Arif, Consultant Gynecologist, Combined Military Hospital, Okara Pakistan

Received: 10 Apr 2020; revised received: 25 Jun 2020; accepted: 30 Jun 2020 coagulation and vasoconstriction leading to end organ ischemia and convulsions ${ }^{4}$.

Eclampsia remains an important cause of maternal and perinatal mortality and morbidity worldwide. Many studies have indicated that over one tenth of maternal deaths in Asia, Africa and about one quarter of maternal deaths in Latin America occur due to eclampsia 5 .

In developed countries its incidence is declining but in developing countries like Pakistan it is a significant contributor to maternal morbidity and mortality ${ }^{6}$. Pakistan is among the six countries where more than $50 \%$ world's maternal deaths occur ${ }^{5}$. According to WHO and UNICEF every year $18 \%$ of maternal deaths are caused by pre-eclampsia and eclampsia and only $40 \%$ of births take place in health facilities in developing countries ${ }^{7}$. The 2007 Health Survey has revealed thateclampsia caused $11 \%$ direct maternal deaths and is the third leading cause of maternal mortality after hemorrhage and infection ${ }^{3}$.

In USA its incidence is $4.3 / 10,000$ deliveries and in UK it is $4.9 / 10,000$ deliveries8. According to one 
study prevalence of pre-eclampsia and eclampsia in Pakistan is around $19 \% 6,4$.

Deaths due to eclampsia are secondary to acute renal failure, cerebrovascular hemorrhage, aspiration pneumonia, pulmonary edema, hemolysis, elevated liver enzymes, low platelet (HELLP) syndrome, disseminated intravascular coagulation (DIC), adult respiratory distress syndrome(ARDS) and cardiac failure ${ }^{3}$.

Perinatal morbidity and mortality varies from 5$11 \%$ in developed countries and up to $40 \%$ in developing countries ${ }^{1}$. It is due to prematurity, low birth weight, birth asphyxia and intra uterine growth retardation ${ }^{8}$.

Magnesium sulphate was introduced in 1925 to control convulsions but it was a Collaborative Trial in 1995 (Magpie trial) that confirmed the efficacy of magnesium sulphate as an anticonvulsant of choice in severe pre-eclampsia and eclampsia ${ }^{9}$. MgSO4 is a potent cerebral vasodilator and acts competitively in blocking the entry of calcium into synaptic endings thus altering neuromuscular transmission. Potential hazards of magnesium sulphate include maternal hypotension, oligouria, loss of patellar reflexes, respiratory depression and cardiac arrest ${ }^{9}$. In developing countries there is limited use of Magnesium sulphate due to apprehension regarding it's side effects. The rationale of study was to determine the efficacy of $\mathrm{MgSO} 4$ in reducing maternal morbidity and mortality in patients presenting with eclampsia.

\section{METHODOLOGY}

This was a prospective observational study. It was conducted in the Department of Obstetrics and Gynecology, Combined Military Hospital Bannu, from Jan 2018 to Sep 2019. A sample size of 134 patients was selected through non-probability consecutive technique. Informed consent was obtained from attendants of every patient presenting with eclampsia. The study protocol was approved by the Ethics Committee of the Hospital (IERC/OBS/ 2019/01). Inclusion criteria were the patients developing eclampsia in second half of pregnancy or within ten days after delivery. Exclusion criteria were patients with fits due to epilepsy, cerebral or any other meta-bolic causes as well as patients presenting more than ten days after delivery.

Management of eclampsia is a multidisciplinary approach, as it requires presence of trained midwife, ICU with fully trained staff along with availability of anesthetist as at times patient may require ventilation support. A detailed history was taken from the atten- dants regarding gestational age or time passed after delivery, number of fits, history of hypertension during pregnancy, proteinuria, swelling of feet, headache, visual disturbances, epigastric pain, vomiting, urinary problems, scotomas, or bleeding per vaginum. A thorough general physical and systemic examination was performed. Blood pressure, pulse, oxygen saturation or temperature was recorded. Edema, jaundice, pallor and reflexes were also checked. Obstetrical examination to determine lie and presentation of fetus, amount of liquor and fetal heart rate was recorded. Vaginal examination was performed to note cervical dilation, effacement and station of presenting part. Ultrasound examination and cardio-tocography was recorded to rule out fetal distress. Mode of delivery (cesarean section or vaginal delivery) was decided according to Bishop Score, maternal and fetal condition.

Convulsions were controlled with 4 grams loading dose of magnesium sulphate (intra-venous) over 20 minutes followed by 1 gram/ hour maintenance infusion. The maintenance infusion was continued for 24 hours after the last fit. Blood pressure was controlled with injection labetalol 10mg IV over 2-3 minutes, followed by injection hydralazine $5 \mathrm{mg}$ IV repeated after every 20 minutes till the blood pressure was controlled. Urinary output, respiratory rate, level of consciousness, presence of patellar reflexes and recurrence of fits was recorded during $\mathrm{MgSO} 4$ infusion. Calcium gluconate $(10 \mathrm{ml}$ of $10 \%$ soln.) was kept in hand in case of $\mathrm{MgSO} 4$ toxicity.

Patient requiring ventilator support was assessed. Frequency of complications like disseminated intravascular coagulation (DIC), acute respiratory distress syndrome (ARDS), HELLP syndrome, accute renal failure (ARF) and death due to intra cranial bleed and pulmonary edema was noted. Statistical analysis was performed with SPSS-22. For quantitative variables like age and parity mean was calculated. For qualitative variables like booking status, locality, ventilation support, ARF, DIC, ARDS, HELLP Syndrome frequencies were measured. Descriptive statistical analysis was done.

\section{RESULTS}

A total of 134 patients had eclampsia constituting $6.8 \%$ of obstetric admissions. Case fatality was 14 $(10.4 \%)$ while $89.55 \%$ of the cases responded well to treatment and survived the event. Mean age of patients was $22 \pm 4.51$ years. Thirty patients $(22.4 \%)$ were booked and 104 patients $(77.6 \%)$ were un-booked. Mostly patients $91 \%$ came from rural area and only $9 \%$ were 
from urban areas with booked pregnancies having regular antenatal visits (figure).

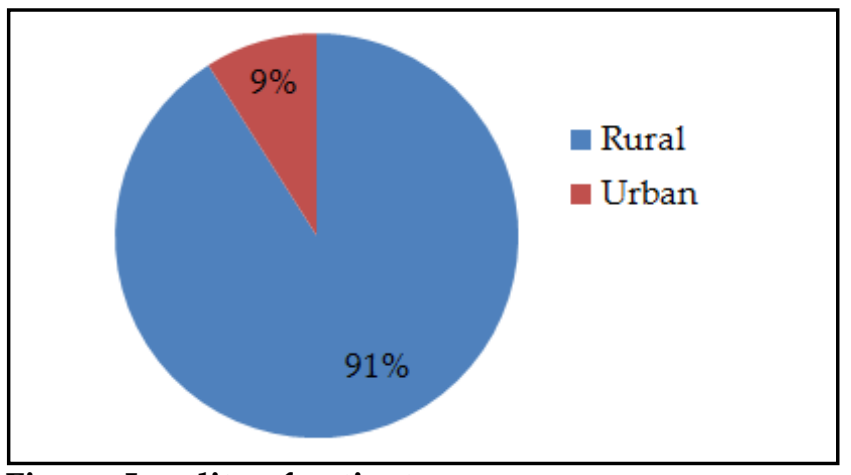

Figure: Locality of patients.

One hundred and twenty six patients with eclampsia presented in antenatal period and only 6 presented in post natal period. $64.9 \%$ underwent cesarean section and $35.1 \%$ delivered vaginally. Maternal mortality due to complications of eclampsia was $6 \%$ due to pulmonary edema while $3.7 \%$ was due to intra-cranial bleed. The frequency of other major complications in our patients is shown in table.

Table: Complications of eclampsia.

\begin{tabular}{l|c}
\hline Complications & n (\%) \\
\hline Ventilator Support & $11(8.2)$ \\
\hline ARF & $46(34.3)$ \\
\hline DIC & $43(32.1)$ \\
\hline ARDS & $35(26.1)$ \\
\hline HELLP & $7(5.2)$ \\
\hline Maternal Mortality & $14(10.4)$ \\
\hline
\end{tabular}

\section{DISCUSSION}

The pathogenesis of eclampsia is unknown, various proposed factors include maternal immunological intolerance, altered placental implantation, vascular endothelial growth factor (VEGF), placental growth factor (PlGF), genetic, nutritional and environmental factors as well as inflammatory changes.

Hypertensive disorders in pregnancy represent wide spectrum of conditions; pre eclampsia, high blood pressure and proteinuria, while eclampsia is the occurrence of one or more generalized convulsions or coma in the presence of pre-eclampsia and in the absence of other neurological conditions. Eclampsia causes increased maternal morbidity and mortality. Once it occurs, hospital inpatient management is required for effective control of convulsions ${ }^{11}$.

In the developing countries eclampsia is the major cause of seizures during pregnancy ${ }^{5}$. The main reason is non-availability of health care facilities especially in the rural areas. In our study the drainage area was mostly rural areas of Miranshah and South Waziristan. $\mathrm{MgSO} 4$ is effective in controlling eclamptic seizures and reducing maternal morbidity and mortality. WHO recommends that as soon as patients with eclampsia develops seizures the pregnancy should be terminated within 12-24 hours and has recommended $\mathrm{MgSO} 4$ to be effective, low priced and safe in the management of severe pre-eclampsia and eclampsia ${ }^{8}$.

The Magpie trial which was initiated in 2002 by UK medical research council in collaboration with WHO was a three year study and included 10,000 pre-eclampsia patients from 33 countries all over the world 9 . In that study pre-eclampsia patients were managed with $\mathrm{MgSO} 4$ and it was concluded to have $58 \%$ decreased risk of eclampsia and $45 \%$ lesser risk of maternal mortality. However MgSO4 has not attainted widespread usage in developing countries like Pakistan as has been discussed in a study by Butt et al, the reason behind the decreased usage of $\mathrm{MgSO} 4$ is lack of public awareness of the drug, lack of adequate training of service provider due to non-availability of $\mathrm{MgSO} 4$ in these areas. Pakistan is a developing country with limited resources in health sector. It has also been mentioned in his study thatits low cost leaves little profit for pharmaceutical companies. Hence there is a supply shortage of this drug in our country ${ }^{12}$.

$\mathrm{MgSO} 4$ was included in National Essential Drug List for treatment of eclampsia and severe pre-eclampsia. In 2007 the Drug List was revised at primary, secondary and tertiary levels for the treatment of eclampsia still Magnesium sulphate non availability as Basic Health Unit, Tehsil Headquarters and District Headquarters is a major issue.

In this study the factors contributing to maternal morbidity and mortality were un-booked status, lack of education, late referral, lack of transport, prolonged coma and multiple seizures prior to admission. This fact is favored by a study done in Madina Hospital, UMDC Faisalabad in 2010 where majority of patients were poor and illiterate ${ }^{13}$. They never had any antenatal visit and presented in coma or after having multiple seizures. In a study by Tabassum et al $65 \%$ patients with eclampsia presented late with major complications due to lack of health awareness, lack of training of traditional birth attendants and financial problems ${ }^{13}$. This fact has also been observed in the study conducted by Butt $\mathrm{A}$ that in Pakistan two third deliveries are still conducted by traditional birth attendants, so 
their role cannot be ignored in reducing maternal morbidity and mortality ${ }^{12}$. Recent demographic and health survey conducted in Pakistan supports the same facts $^{14}$. So education at basic health care level is required regarding efficacy, dosage, toxicity and antidote of $\mathrm{MgSO} 4$.

The reported data in Pakistan showed 18-23\% maternal mortality ${ }^{6}$. In our study, it was $10.4 \%$ as compared to $24 \%$ in a study conducted by Shaikh and Rathore et al. Shaikh et al concluded $19.68 \%$ case fatality rate, which was mainly due to pulmonary edema $(66 \%)^{5}$. Study conducted by Munro et al concluded cerebral hemorr-hage as the main cause of death ${ }^{1}$.

The high perinatal mortality was mostly due to prematurity, which was also supported by other studies conducted in Lahore, Faisalabad, India and Africa $^{2}$. Such neonates are prone to develop bronchopulmonary dysplasia, intra cranial hemorrhage, sepsis and respiratory distress secondary to surfactant defiency ${ }^{15}$. Long term follow up for these babies is required to look for sequel of prematurity ${ }^{16-20}$.

Need for strengthening of intensive care units, trained ICU team for central venous line insertion andfluid monitoring is required in hospitals. Further study and data collection is required to access the barriers to the use of magnesium sulphate in prevention and treatment of Eclampsia. Further study should be conducted regarding perinatal outcomes and follow up to detect long term sequel of pre maturity and birth asphaxialike cerebral palsy ${ }^{21-25}$.

\section{CONCLUSION}

Eclampsia is a serious and life threatening cause of maternal morbidity and mortality in obstetrics. Magnesium sulphate is a safe, cost effective and time tested drug for prevention and treatment of eclampsia. Early booking and regular antenatal checkups should be encouraged. Prevention of eclampsia still remains a big challenge in developing countries like Pakistan.

\section{CONFLICT OF INTEREST}

This study has no conflict of interest to be declared by any author.

\section{REFERENCES}

1. Munro PT. Management of eclampsia in the accident and emergency department. J Accid Emerg Med 2000; 17(1): 7-11.

2. Mahler $\mathrm{H}$. The safe motherhood initiative a call to action. Lancet 1987; 329(8534): 668-70.

3. Verma K, Baniya GC, Agrawal S, Lomrod S. A study of maternal and perinatal outcome in eclampsia patients. Indian J of Obstet Gynecol Res 2016; 3(4): 318-21.

4. Pannu D, Das B, Hazari P. Maternal and perinatal outcome in eclampsia and factors affecting the outcome: a study in North
Indian population. Int J of Reprod Contracept Obstet Gynecol 2014; 3(2): 347-51.

5. Shaikh F, Abbas S, Balouch I. Frequency and outcome of eclampsia. Gomal J Med Sci 2016; 14(4): 200-02.

6. Rathore R, Butt NF, Iqbal A. Complications and outcome of patients of pre-eclampsia and eclampsia presenting to medical wards of Mayo Hospital Lahore. Annals 2010; 16(1): 17-19.

7. Kanwal M, Iftikhar PM. Role of magniseum sulphate is an effective therapy for treatment and prophylaxis of seizures in preeclampsia and eclampsia. ISRA Med J 2016; 8(1): 7-10.

8. Tariq M, Rehman H, Tayyab M. Clinico-pathological study of pre-eclampsia. Biomedical 2000; 16(7): 60-65.

9. Tukur J. The use of magnesium sulphate for treatment of severe pre-eclampsia and eclampsia. Annals African Med 2009; 8(2): 7680.

10. WHO. Coverage of maternity care. A list of available information. Geneva, Switzerland: Maternal and newborn health/safe motherhood. 1997 Avalibal at Internat. https://apps.who.int/ iris/handle/10665/63878.

11. Duhig K, Vandermolen B, Shennan A. Recent advances in the diagnosis and management of pre-eclampsia. F1000 Res 2018; 7(1): 242-45.

12. Butt AI. Magnesium sulphate for prevention and treatment of pre-eclampsia and eclampsia in Pakistan. J Soc Obstet Gynaecol 2013; 3(2): 107-19.

13. Tabassum N, Umber A, Khan S. Eclampsia: A major cause of feto-maternal mortality and morbidity. Annals KEMU 2010; 16(3): 202-05.

14. National institute of Population Studies and Macro international Inc., Pakistan Demographic and Health Survey, 2006-07 (Islamabad: Avalibalat Internet). https://scholar.google.com/scholar? $\mathrm{hl}=$ en\&as_sdt $=0 \% 2 \mathrm{C} 5 \& \mathrm{q}=$ Pakistan + Demographic + and + Health + Survey \% 2C+\& btnG.

15. Praveenkumar AM, Patil R, Pachpande V. Materna and fetal outcome in Eclampsia. Ann. Int Med Den Res 2017; 3(2): 01-06.

16. Sarkar M, Basak S, Mondal SK, Das S, Roy D, Mondal J. Pre eclampsia and cardiovascular disease. Int J of Reprod Contracpt Obstet Gynecol 2014; 3(3): 653-55.

17. Nessa K, Dewan F, Parvin T, Nahrin N. Simplification of loading dose of $\mathrm{MgSO} 4$ in the management of eclampsia. Bangladesh J Obstet Gynecol 2015; 30(2): 67-73.

18. Jain R, Bindal J. Maternal and perinatal outcomes in eclampsia: a retrospective analysis in a referral hospital. Int J of Reprod, Contraception, Obstet Gynecol 2017; 6(7): 2806-11.

19. Nobis PN, Hajong A. Eclampsia in India through the decades. J Obs Gyncol India 2016; 66(Suppl-1): 172-76.

20. Bernstein PS. National partnership for maternal safety: Consensus bundle on severe hypertension during pregnancy and the postpartum period. Obstet Gynecol 2019; 133(6): 1287-90.

21. Tooher J, Thornton C, Markis A, Ogle R. All hypertensive disorders of pregnancy increase the risk of further cardiovascular disease. Hypertension 2017; 70(5): 798-03.

22. Omu A.E, Harmi J, Vedi H. Magnesium Sulphate therapy in women with Pre-Eclampsia and Eclampsia in Kuwait. Med Princ Pract 2008; 17(1): 227-32.

23. Y. Ni and W. Cheng. Comparisons of indications of pregnancy termination and prognosis of mothers and neonates in early and late onset pre-eclampsia. Hypertens Preg 2016; 35(3): 315-322.

24. Nguejack CT. Comparison of materno-fetal predictors and short term outcomes between early and late onset pre-eclampsia in the low income setting of Douala Cameroon. International Journal of Gynecology and Obstetrics. 2019; 142(2): 228-34.

25. Dag ZO, Isik Y, Simsik Y. Atypical eclampsia and postpartum status epilepticus. Pan African Med J 2015; 9(2): 257-9. 